\section{Acute Pancreatitis and Acute Cholecystitis \\ Caused by Hemobilia after Percutaneous Ultrasound-Guided Liver Biopsy}

We report here a patient who suffered three complications after ultrasoundguided percutaneous liver biopsy: hemobilia, acute biliary pancreatitis, and acute cholecystitis. Endoscopic sphincterotomy and cholecystectomy were successfully carried out, allowing a minimally invasive approach to manage these events.

A 21-year-old woman underwent an ultrasound-guided liver biopsy as part of a preoperative kidney transplantation protocol. Her background included chronic renal failure due to glomerulonephritis and hepatitis $C$. One hour after the procedure, she experienced acute epigastric pain and melena, without hemodynamic compromise. Her hemoglobin level decreased from $8.9 \mathrm{~g} / \mathrm{dl}$ to $7.7 \mathrm{~g} / \mathrm{dl}$ (normal range $13.5-15.0 \mathrm{~g} / \mathrm{dl}$ ). An ultrasound examination demonstrated free peritoneal fluid and a large quantity of echogenic material in the gallbladder. The following day, she developed nausea, vomiting, worsening pain, and jaundice. Serum amylase was $600 \mathrm{U} / \mathrm{l}$ (normal: below $130 \mathrm{U} / \mathrm{l}$ ), total bilirubin $11.5 \mathrm{mg} / \mathrm{dl}$ (normal: $0.2-1.0 \mathrm{mg} /$ dl). She received three units of packed red blood cells. Computed tomography and magnetic resonance cholangiopancreatography (MRCP) revealed edematous pancreatitis, a dilated gallbladder, and clots inside the common bile duct and gallbladder. Endoscopic retrograde cholangiopancreatography confirmed that there were blood clots obstructing the major duodenal papilla (Figure $\mathbf{1}$ ), and the cholangiogram demonstrated irregular filling defects throughout the bile ducts and gallbladder (Figure 2). Endoscopic sphincterotomy was carried out, and a large and hypertensive amount of fresh blood clot was drained with a basket and a balloon catheter. A hepatic angiogram did not show any evidence of further active bleeding. The patient's condition and jaundice initially improved, but 5 days later, recurrent ab-

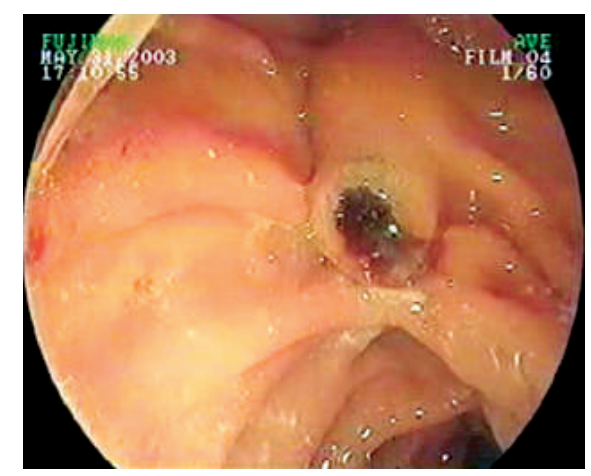

Figure 1 An impacted clot in the major duodenal papilla.

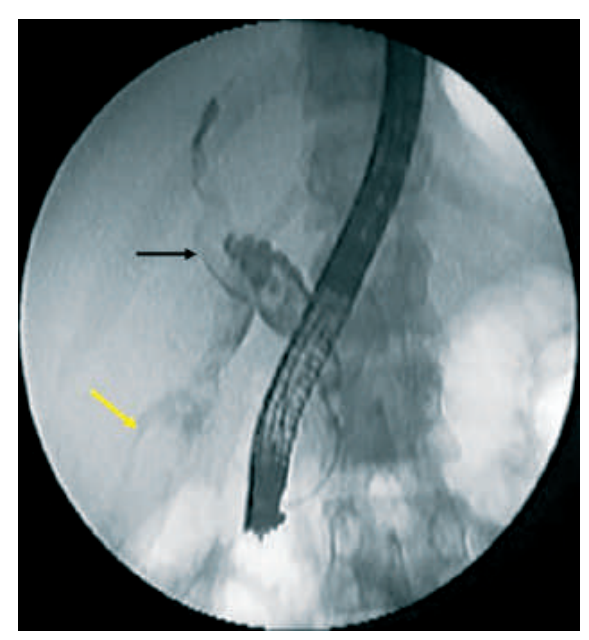

Figure 2 Clots inside the common bile duct (black arrow) and gallbladder (yellow arrow).

dominal tenderness over the right upper quadrant, fever, and leukocytosis were noted. Ultrasound revealed a distended gallbladder containing echogenic materials. A cholecystectomy was performed, confirming blood clots and purulent fluid inside the gallbladder. The patient was discharged home after a course of antibiotics, and has remained well in the last 18 months.

\section{Video}

Video sequence may be viewed online: http://www.thieme-connect.com/DOI/ DOI?10.1055/s-2005-870407
The average time observed between a liver biopsy and the onset of hemobilia is approximately 5 days [1]. Angiography can be useful both to confirm the diagnosis and to attempt treatment by embolizing the bleeding vessel [2]. Ultrasound can detect clots in the dilated bile ducts and gallbladder. MRCP shows a negative magnetic signal in the biliary duct and gallbladder, corresponding to blood clots. When the blood clots completely obstruct the duodenal papilla, as observed in the patient described here, jaundice and biliary pancreatitis may subside; this observation has also been reported by other authors $[3,4]$. Acute cholecystitis has also been described after liver biopsy [5]. In the present case, the distended gallbladder full of large blood clots was not able to empty itself even after sphincterotomy and wide biliary drainage, and cholecystectomy was necessary to manage this complication.

\section{W. Albuquerque', V. Arantes',} K. de Paula Farah ${ }^{2}$, J. R. Lambertucci ${ }^{3}$

${ }^{1}$ Endoscopy Unit, Alfa Institute of Gastroenterology, University Hospital,

Federal University of Minas Gerais, Minas Gerais, Brazil

${ }^{2}$ Dept. of Nephrology, Faculty of Medicine, Federal University of Minas Gerais, Minas Gerais, Brazil

${ }^{3}$ Dept. of Internal Medicine, Faculty of Medicine, Federal University of Minas Gerais, Minas Gerais, Brazil 


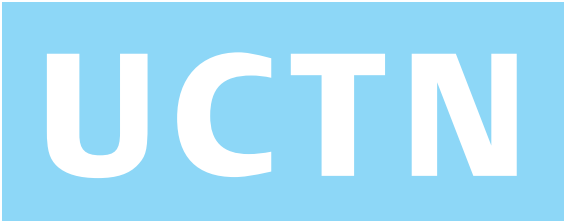

References

${ }^{1}$ Merrell SW, Schneider PD. Hemobilia: evolution of current diagnosis and treatment. West J Med 1991; 155: 621-625

${ }^{2}$ Lichtenstein DR, Kim D, Chopra S. Delayed massive hemobilia following percutaneous liver biopsy: treatment by embolotherapy. Am J Gastroenterol 1992; 87: $1833-1888$

${ }^{3}$ Kim HJ, Shin JH, Lee YYet al. Hemobilia causing acute biliary pancreatitis after percutaneous liver biopsy. Endoscopy 1999; 31: S18-S19
${ }^{4}$ Sood A, Midha V, Kumar A, Sood N. Acute pancreatitis with hemobilia following percutaneous liver biopsy. Indian J Gastroenterol 2002; 21: 81 - 82 ${ }^{5}$ Coelho JC, Bonin EA, da Costa MAet al. Acute cholecystitis secondary to hemobilia after percutaneous liver biopsy. Dig Surg 2001; 18: 227-229
Corresponding Author

\section{W. Albuquerque, M.D.}

Endoscopy Unit, Alfa Institute of Gastroenterology, University Hospital, Federal University of Minas Gerais Alameda Centauro, 298,

Ville de Montagne Nova Lima, Minas Gerais Brazil

Fax: $\quad+55-31-33397513$

E-mail: waltonendoscopia@uol.com.br 